\title{
Medición de la Madurez de La Gestión del Conocimiento en la Escuela de Ciencias Básicas TeCnOLogía E InGeniería de LA UnAd
}

\author{
Measurement of the Maturity of Knowledge \\ Management in the Basic Sciences Technology \\ AND ENGINEERING SCHOOL OF UNAD
}

'Luis Montañez Carrillo, ²Jenny Paola Lis Gutiérrez

${ }^{1}$ Universidad Nacional Abierta y a Distancia UNAD, Escuela de Ciencias Básicas, Tecnología e Ingeniería, Bogotá, Colombia.

${ }^{2}$ Fundación Universitaria Konrad Lorenz, Centro de Investigaciones de la Escuela de Negocios, Bogotá, Colombia.

\footnotetext{
${ }^{1}$ luis.montanez@unad.edu.co

${ }^{2}$ jenny.lis@konradlorenz.edu.co
}

Recibido: 01/09/2015 • Aprobado: 12/11/2015

\section{RESUMEN}

El objetivo de este trabajo es medir la madurez de la Gestión del Conocimiento en la Escuela de Ciencias Básicas, Tecnología e Ingeniería (ECBTI) de la Universidad Nacional Abierta y a Distancia, Unad (Colombia). Para este propósito, se adaptó el modelo denominado General Knowledge Management Maturity Model (G-KMMM) [1], el cual propone 5 niveles de madurez: inicial, conciencia, definido, gestionado y optimizado, y establece 3 áreas clave: personas, procesos y tecnología. La modificación del modelo consistió en adicionar un área clave denominada estrategia. Para el estudio se aplicó un instrumento de evaluación que proporciona el modelo G-KMMM, a 307 colaboradores de la ECBTI. En relación con los resultados se encontró que el nivel de madurez del área clave personas era 2 (conciencia), del área clave procesos era 3 (definido), del área clave tecnología era 5 (optimizado) y del área clave estrategia era 5 (optimizado). Con respecto al nivel de madurez de GC global de la ECBTI se encontró que era 4 (gestionado).

Palabras clave: gestión del conocimiento, modelos de madurez, niveles de madurez, educación, instituciones de educación superior.

\section{Abstract}

The objective of this study is to measure the maturity of Knowledge Management in the Basic Sciences, Technology and Engineering School (ECBTI) of the Open and Distance National University (UNAD, Colombia). For this purpose, it was adapted the model called General Knowledge Management Maturity Model (G-KMMM) [1], which offers 5 levels of maturity: initial, conscience, defined, managed and optimized, and sets 3 key areas: 
people, processes and technology. The modification of the model consisted of adding a key area named strategy. For the study it was applied an assessment tool that provides the model G-KMMM, to 307 collaborators of the ECBTI. In relation to the results, it was found that the level of maturity of the key area people was 2 (consciousness), of the key area processes was 3 (defined), of the key area technology was 5 (optimized) and of the key area strategy was 5 (optimized). With regard to the global maturity level of ECBTI, it was found to be 4 (managed).

Keywords: education, knowledge management, maturity levels, maturity models.

\section{INTRODUCCIÓN}

Este artículo tiene como propósito presentar un estudio que se llevó a cabo para medir la madurez de la Gestión del Conocimiento (GC) en la Escuela de Ciencias Básicas, Tecnología e Ingeniería (ECBTI) de la Universidad Nacional Abierta y a Distancia (Unad). Para la realización de la medición se adaptó el modelo de madurez de GC denominado General Knowledge Management Maturity Model (G$K M M M$ ) [1]. A las tres áreas clave del modelo (personas, procesos y tecnología), se le adicionó una cuarta: estrategia, lo que permitió realizar la medición de madurez de manera agregada y específica para las áreas anteriormente mencionadas.

Inicialmente, es importante indicar que en el presente trabajo de investigación, el conocimiento se concibe como el producto de las experiencias, habilidades y aprendizaje de un individuo pawra realizar una acción de manera eficiente. En cuanto al contexto organizacional, el conocimiento se constituye como información que brinda la posibilidad de generar acciones que contribuyen al desarrollo de la organización. Por otra parte, la Gestión del Conocimiento se entiende como el proceso mediante el cual se crea, captura, transforma y usa el conocimiento orientado al desarrollo organizacional y a la creación de ventajas competitivas. La Gestión del Conocimiento en el contexto de las Instituciones de Educación Superior se concibe como el proceso a través del cual se crea, adquiere, procesa, conserva, transmite y transfiere el conocimiento, mediante la articulación de la docencia, la investigación y la extensión con el fin de formar profesionales que posean el conocimiento para atender las necesidades sociales, científicas y técnicas de la sociedad.
En cuanto a los modelos de madurez de GC, se comprenden como mecanismos que permiten medir y comparar las iniciativas que emprende una organización en aras de gestionar el conocimiento que en ella se crea. Estos modelos permiten tanto establecer el estado actual en el que se encuentra una organización en materia de GC como generar una ruta para mejorar ese estado actual. En esta medida se establecen como guías para la implementación de la GC en etapas y contemplan el tránsito gradual de un estado inicial hasta uno óptimo.

En relación con el modelo de madurez seleccionado para realizar el diagnóstico, es decir, el modelo G-KMMM, cabe señalar que define 3 áreas clave que agrupan las capacidades y prácticas de GC que desarrolla una organización. Las 3 áreas clave que propone el modelo son: personas (comportamientos, habilidades, sistemas de incentivos y estructura organizacional que existe para crear y compartir el conocimiento), procesos (como se crea, almacena, comparte y usa el conocimiento) y tecnología (sistema empleado para el intercambio de conocimiento). Como se mencionó anteriormente, en este trabajo de investigación se implementó un área clave denominada estrategia, debido a que se requería conocer cómo se alinean los esfuerzos de la GC con los focos estratégicos de la ECBTI, considerando que el conocimiento es un recurso intangible que tiene un gran valor para las Instituciones de Educación Superior.

Adicionalmente, el modelo propone 5 niveles de madurez que categorizan los distintos estados en materia de GC de cada una de las áreas clave. Los 
niveles de madurez de GC del modelo G-KMMM son: inicial, conciencia, definido, gestionado/establecido y optimizado. En el nivel inicial, la organización tiene poca (o ninguna) intención de usar el conocimiento; en el nivel conciencia, la organización es consciente y tiene la intención de GC pero posiblemente no sepa cómo; en el nivel definido, la organización ha puesto en marcha una infraestructura básica que soporta la GC; en el nivel gestionado/establecido, las iniciativas de GC están plenamente establecidas en la organización y en el nivel optimizado, la GC está plenamente integrada a la organización y sometida a procesos de mejoramiento continuo.

Ahora bien, la Unad es un ente universitario autónomo del orden nacional, con régimen especial, personería jurídica, autonomía académica, administrativa y financiera, patrimonio independiente y capacidad para gobernarse [2]. La misión de esta Universidad es contribuir a la educación para todos a través de la modalidad abierta y a distancia y en ambientes virtuales de aprendizaje. Utiliza las tecnologías de la información y de las comunicaciones (TIC) para fomentar y acompañar el aprendizaje autónomo. Cuenta con más de 60 centros de formación distribuidos en todo el territorio de Colombia, por lo cual una gran cantidad de sus programas de formación tienen cobertura nacional [3]. A partir del año 2008, la Unad implementó el Sistema Integrado de Gestión (SIG), el cual tiene como objetivo impulsar la GC y la innovación a partir del incentivo a la producción intelectual con calidad y pertinencia [4].

En el marco de los ámbitos de actuación y contextos para la formación del estudiante, la Unad cuenta con Escuelas, las cuales cumplen funciones equivalentes a las de las Facultades en otras Instituciones de Educación Superior: gestionan los procesos académicos correspondientes a los programas de formación de la Universidad (de acuerdo con la disciplina o campo de conocimiento); diseñan, desarrollan, validan y actualizan los planes curriculares de los programas; aplican las políticas, directrices y estrategias definidas para la consolidación académica; coordinan los planes, programas y proyectos y lideran la función investigativa, entre otras.
La Escuela de Ciencias Básicas, Tecnología e Ingeniería (ECBTI) contaba en el primer semestre de 2016 con una población de 570 personas distribuidas en todo el territorio nacional y más de 13.000 estudiantes matriculados en 15 programas de pregrado y posgrado [5]. Esta Escuela hace uso de las TIC para gestionar el conocimiento tanto académico como organizacional que se crea en cada uno de los centros de formación en el ámbito nacional. De acuerdo con [6], las TIC permiten inventariar y acumular el conocimiento que se desarrolla para su posterior utilización, ofrecen herramientas que permiten acercarse al conocimiento, facilitan la comunicación y proporcionan un medio para la generación de nuevos conocimientos.

En este orden de ideas, en IES como en la Unad donde se ha planteado como objetivo impulsar la Gestión del Conocimiento (GC) y la innovación, se hace necesario desarrollar investigaciones que permitan conocer en qué estado se encuentran las Escuelas en materia de madurez de Gestión de Conocimiento. Esto con el fin de que en posteriores estudios se propongan planes de mejoramiento que permitan mejorar su nivel de madurez.

Por ello, el objetivo general de la presente investigación es diagnosticar la madurez de la GC en la Escuela de Ciencias Básicas, Tecnología e Ingeniería ECBTI de la Unad. En relación con los objetivos específicos se pretende:

- Realizar una revisión documental sistemática sobre los modelos para medir la madurez de GC.

- Elaborar un análisis comparativo para la selección de un modelo para medir la madurez de GC que pueda ser aplicado en la ECBTI.

- Identificar el nivel de madurez de GC tanto global como por áreas clave de la ECBTI.

En cuanto a la metodología, se trata de una investigación descriptiva y evaluativa que se elaboró mediante un enfoque cuantitativo. Para esto último, se realizó la medición de la madurez de la GC a través de un instrumento tipo encuesta que proporcionó el modelo G-KMMM, el cual define cinco 
(5) niveles de madurez (inicial, conciencia, definido, gestionado y optimizado) y tres (3) áreas clave (personas, procesos y tecnología). Para complementar el diagnóstico se implementó en este trabajo el área clave estrategia, que permitió comprender la forma como se alinean los esfuerzos de la GC con los focos estratégicos de la ECBTI [7].

La encuesta se aplicó mediante un formulario de Google y se envió por el correo institucional de la Unad a la población de la ECBTI conformada por 570 personas. La muestra obtenida en el diligenciamiento del instrumento fue de 307 personas. Posteriormente, se analizó la información obtenida y se identificó el nivel de madurez de la ECBTI tanto de manera global como por áreas clave.

\section{Marco de Referencia}

En este apartado se presenta una revisión de la literatura que aborda el concepto de GC y de los modelos de madurez de GC.

\section{A. Gestión del conocimiento (gc)}

De acuerdo con [8], la GC es un conjunto de procesos sistemáticos en los que se identifica, capta, trata, desarrolla, comparte y se utiliza el conocimiento, para ser orientado al desarrollo organizacional y a la generación de una ventaja competitiva para la organización.

Para [9], la GC incorpora procesos sistemáticos para encontrar, seleccionar, organizar y presentar la información de tal manera que mejore tanto la comprensión de los empleados como el uso de los bienes de la compañía. Por su parte, [10] establecieron que la GC, como su nombre lo indica, gestiona la creación, captura, transformación y uso del conocimiento; su principal función es planificar, implementar, operar y administrar las actividades intrínsecas del conocimiento y los programas requeridos para la gestión efectiva del capital intelectual.

[11] afirmaron que la GC se puede concebir como una forma de dirección encaminada a la maximización del rendimiento del capital intelectual de una organización. Los autores mencionan que el capital intelectual se refiere al conjunto de competencias organizacionales distintivas, de carácter intangible, que brindan la posibilidad de crear ventajas para la obtención de presupuestos a través de la colaboración y compromiso de los miembros de la organización y el uso de procesos en la producción, transmisión y transferencia de un conocimiento innovador.

\section{B. Las instituciones de educación superior (ies) y la gc}

Una diferencia fundamental entre las organizaciones del sector productivo y las IES, radica en que las primeras se preocupan principalmente por la gestión humana y de recursos. Por el contrario, en las IES la preocupación gira en torno a modelos de conocimiento; las IES crean conocimiento, forman profesionales y transfieren conocimientos científicos o técnicos que permiten resolver problemas y satisfacer las necesidades de la sociedad. Asimismo, dividen los saberes con base en criterios teóricos o del ámbito profesional y se someten a múltiples evaluaciones para certificar el proceso de transmisión de conocimientos que se lleva a cabo [12], [13].

Las IES tienen entonces múltiples retos en la sociedad del conocimiento: renovar los sistemas económicos y sociales, ampliar el conocimiento y las habilidades, participar efectivamente en la producción de conocimiento, tener una relación directa con el sector productivo, los centros de investigación y otras instituciones, además de brindar a la sociedad profesionales de primer nivel. Las universidades se conciben como el principal instrumento de la sociedad para la constante búsqueda del conocimiento [14], [15], [16].

[17] estableció que las IES se presentan como sistemas sociales en los cuales se crea, adquiere, procesa, conserva, transmite y transfiere el conocimiento. Lo anterior, mediante estructuras complejas que articulan la docencia, la investigación y la extensión con el fin de formar ciudadanos que posean el conocimiento para desempeñarse profesionalmente en diferentes disciplinas y que sean capaces de resolver problemas sociales, científicos 
y técnicos que se presentan en la sociedad. En este orden de ideas, en las IES es posible encontrar que:

- La reproducción del conocimiento se efectúa a través de la docencia, la investigación y la extensión.

- Las IES invierten en formación y desarrollo de su personal (formación continua).

- El conocimiento está en las personas.

- La mayoría de las personas no tienen contabilizado su conocimiento ni su capital intelectual.

En esta medida, la GC en las Instituciones de Educación Superior pretende integrar los recursos humanos, procesos académicos y avances tecnológicos involucrados en el diseño, la captura y ejecución de la infraestructura intelectual de una institución educativa. El enfoque se apoya en la elaboración y la gestión académica para aprender manteniendo el equilibrio entre las diversas entidades en un medio ambiente académico [10].

Según [18], para que las universidades puedan mejorar los procesos de GC, deben entender inicialmente lo que constituye el conocimiento, es decir, lo que deben gestionar. Luego se debe evaluar la situación actual de la GC lo cual debe considerarse como un punto de partida. La evaluación es el primer paso hacia la mejora; no se puede mejorar lo que no se puede medir bien sea de manera formal o informal. De allí, la importancia del presente trabajo.

\section{Modelos de madurez de gestión del conoci- miento (mmgc)}

Los Modelos de Madurez de Gestión de Conocimiento (MMGC) se basan en la necesidad de tener una hoja de ruta para cualquier organización que comienza a implementar la GC. Brindan una visión clara con una descripción del camino por seguir. Un MMGC puede proporcionar una comprensión común de los términos y elementos involucrados en la implementación de GC [19].

Desde el punto de vista de [7], en las últimas dos décadas, el conocimiento ha empezado a tener más importancia en el ámbito empresarial, debido a que es el recurso que mayor incidencia tiene sobre el desempeño organizacional y la creación de ventajas competitivas. A partir de esta premisa, autores como Paulzen, Doumi, Perc \& Cereijo-Roibas a principios del siglo XXI empezaron a articular la GC con los modelos de madurez que surgieron de la ingeniería de software y, de este modo, se estructuraron las primeras guías para la implementación de la GC en etapas, que contemplan el tránsito gradual de un estado inicial hasta uno óptimo. La estructura básica de los MMGC desde la perspectiva de [7] es la siguiente:

- Áreas clave. Agrupan las capacidades y prácticas de GC que desarrolla una organización, de acuerdo con la articulación que tengan con elementos como la tecnología, la cultura y los procesos, entre otros.

- Escala de madurez. Regularmente, los modelos de este tipo constan de 5 niveles que indican los distintos estados de las capacidades y prácticas en materia de GC, partiendo de un nivel principiante hasta llegar a un máximo de desarrollo y consolidación de la organización.

\section{Metodología}

En cuanto a la metodología, se trata de una investigación descriptiva que se elaboró mediante un enfoque cuantitativo. En materia del diseño de investigación, se definieron una serie de etapas concebidas para responder a la pregunta de investigación y alcanzar los objetivos planteados. En la primera etapa denominada Estado del arte se realizó una revisión de la literatura en el ámbito académico en materia de modelos para medir la madurez de GC, por medio de búsquedas en bases de datos académicas tanto estructuradas como no estructuradas, de trabajos de investigación y artículos sobre el tema objeto de estudio. Dentro de la revisión de literatura realizada en regiones como América del Norte, América Latina, Europa, Asia y África, se encontraron 24 modelos para medir la madurez de GC en organizaciones. En la tabla 1 se presenta una relación de dichos modelos: 
TABLA I.

Modelos Para Medir la Madurez de GC Encontrados en el Estado del Arte

\begin{tabular}{|c|c|c|}
\hline MODELO & AUTOR(ES) & AÑO \\
\hline Knowledge Process Quality Model (KPQM). & Paulzen, Doumi, Perc \& Cereijo-Roibas & 2002 \\
\hline Organizational Self-Assessment of Knowledge Management Maturity. & Kulkarni \& St Louis & 2003 \\
\hline $\begin{array}{l}\text { Diagnóstico de la gestión de conocimiento en una empresa grande de } \\
\text { Barranquilla (Colombia). }\end{array}$ & González, Castro \& Roncallo & 2004 \\
\hline Strategic knowledge management maturity model. & Snyman \& Kruger & 2005 \\
\hline A Knowledge Management Maturity Model and Application. & Feng & 2006 \\
\hline $\begin{array}{l}\text { Gartner: A Knowledge Management Maturity Model Explains Where You 're } \\
\text { Going and How to Get There. }\end{array}$ & Harris & 2006 \\
\hline Modelo general de madurez de GC (G-KMMM) & Teah, Pee \& Kankanhalli & 2006 \\
\hline The Support Center Maturity Model. & Joslin & 2007 \\
\hline Knowledge management profile maturity model. & $\begin{array}{l}\text { Gaál, Szabó, Kovács, Obermayer- } \\
\text { Kovács \& Csepregi }\end{array}$ & 2008 \\
\hline Knowledge navigator model (KNM). & Hsieh, Lin \& Lin & 2009 \\
\hline Karagabi KM Model. & González, Joaquí \& Collazos & 2009 \\
\hline $\begin{array}{l}\text { Estudios de caso sobre la GC en cuatro organizaciones colombianas líderes } \\
\text { en penetración de mercado. }\end{array}$ & Briceño \& Bernal & 2010 \\
\hline Facilitating new knowledge creation and obtaining KM maturity. & Arling \& Chun & 2011 \\
\hline $\begin{array}{l}\text { Knowledge Management } \\
\text { Maturity Model: An Engineering Approach. }\end{array}$ & $\begin{array}{l}\text { Kuriakose, Raj, Satya Murty \& } \\
\text { Swaminathan }\end{array}$ & 2011 \\
\hline KM3 & Oliveira, Pedron, Romão \& Becker & 2011 \\
\hline $\begin{array}{l}\text { Análisis de madurez de la gestión del capital intelectual en la pequeña y } \\
\text { mediana empresa latinoamericana }\end{array}$ & Romero \& Pascual & 2011 \\
\hline La GC en Instituciones de Educación Superior (IES) de Medellín. & Uribe & 2013 \\
\hline $\begin{array}{l}\text { Análisis comparativo de los modelos de madurez de la GC en grandes } \\
\text { empresas de Medellín-Colombia. }\end{array}$ & Durango \& Pérez & 2013 \\
\hline $\begin{array}{l}\text { Metodología para evaluar la madurez de la GC en algunas grandes empresas } \\
\text { colombianas. }\end{array}$ & Durango, Quintero \& Ruiz & 2013 \\
\hline $\begin{array}{l}\text { Madurez de la GC y la gestión de procesos en empresas que utilizan la } \\
\text { plataforma virtual Talentum. }\end{array}$ & Acuña, Gómez, \& Pintor & 2014 \\
\hline Knowledge Management Maturity in Construction Companies. & Wibowo \& Waluyo & 2015 \\
\hline $\begin{array}{l}\text { Knowledge Management Maturity Level in a Brazilian Air Force Flight Test } \\
\text { Environment. }\end{array}$ & Follador & 2015 \\
\hline $\begin{array}{l}\text { Exploring knowledge management maturity from funcionalist and interpretivist } \\
\text { perspectives. }\end{array}$ & Arias-Pérez \& Durango-Yepes & 2015 \\
\hline $\begin{array}{l}\text { Construcción de un MMGC para una multinacional de alimentos de una } \\
\text { economía emergente. }\end{array}$ & $\begin{array}{l}\text { Arias-Pérez, Tavera-Mesías } \\
\text { \& Castaño-Serna }\end{array}$ & 2016 \\
\hline
\end{tabular}


Posteriormente, en la segunda etapa denominada Selección del modelo para medir la madurez de GC se llevó a cabo un análisis comparativo con los 24 modelos encontrados para seleccionar el más apropiado. En la elaboración del análisis comparativo se definieron una serie de criterios de comparación relacionados con el número de niveles de madurez del modelo, las áreas clave que establece, si define un instrumento de medición, la brevedad de dicho instrumento, el idioma del modelo, la complejidad para su aplicación, la adaptabilidad del modelo en diferentes contextos y su libre uso.

Con estos criterios de comparación se realizó el análisis para identificar las debilidades y fortalezas de los modelos encontrados en la revisión de literatura. En este orden de ideas, partiendo de dicho análisis, se procedió a desarrollar una evaluación de orden cuantitativa, para la selección del modelo, asignando una calificación de 0 a 1 a cada criterio. El modelo para medir la madurez de GC con mayor calificación fue el Modelo general de madurez de GC (G-KMMM) [1], el cual tuvo una puntuación de 7 .

En este sentido, era el modelo con mayores fortalezas y mejores características para la realización del diagnóstico de madurez de GC en la ECBTI. Adicionalmente, vale la pena resaltar que el modelo G-KMMM ha sido un referente en materia de modelos de madurez de GC ya que se ha empleado como base para la construcción de una gran cantidad de modelos y metodologías tanto internacionales como nacionales, para medir la GC.

Una de las fortalezas más representativas del modelo G-KMMM, tiene que ver con el hecho de que se puede aplicar a diferentes objetos de análisis, incluidos la organización como conjunto y de manera individual para sus respectivas unidades. Adicionalmente, el modelo brinda una explicación muy detallada del instrumento de medición para proporcionar un enfoque sistemático y estructurado que garantiza la transparencia del procedimiento de evaluación. Es importante señalar que el modelo GKMMM adopta una estructura organizada y define claramente cada nivel de madurez y sus áreas clave, así como sus respectivas características [1].

De acuerdo con [20], el modelo G-KMMM propone tres áreas clave:

Personas y organización. Constituye la confianza (fe en las intenciones y comportamientos de la organización), las habilidades T-shapped (grado de comprensión de las tareas propias y de los demás), los sistemas de incentivos (recompensas económicas o simbólicas para motivar a los empleados a crear y compartir el conocimiento) y la estructura organizacional (procedimientos, políticas y estándares).

Procesos. Se refiere a la forma en cómo se crea (desarrollo de nuevos conocimientos y procedimientos), recopila (adquisición y registro de datos, información y conocimiento), comparte (intercambio de mejores prácticas) y usa el conocimiento (creación de valor para la organización).

Tecnología. Se concibe como el sistema que se emplea para el intercambio de conocimiento en toda la organización. Esta área clave está comprendida por los servicios de GC (aplicaciones que usa la empresa), la infraestructura tecnológica (inversión en TIC para soportar las actividades de GC) y actitud hacia las TIC (disposición de las personas frente a las tecnologías que apoyan las iniciativas de GC).

De acuerdo con [21], cada nivel de madurez está conformado por las 3 áreas clave y cada área clave está descrita por una serie de características comunes. Dichas características especifican las prácticas principales que cuando son direccionadas colectivamente, contribuyen a alcanzar los objetivos de un área clave. En la tabla 2 se presenta la estructura del modelo G-KMM. 
TABLA ॥

Estructura del Modelo G-Kmmm de Teah, Pee \& Kankanhalli (2006) [20]

\begin{tabular}{|c|c|c|c|c|}
\hline \multirow{2}{*}{$\begin{array}{l}\text { NIVEL DE } \\
\text { MADUREZ }\end{array}$} & \multirow[b]{2}{*}{ DESCRIPCIÓN } & \multicolumn{3}{|c|}{ ÁREAS CLAVE } \\
\hline & & $\begin{array}{l}\text { PERSONAS Y } \\
\text { ORGANIZACIÓN }\end{array}$ & PROCESOS & TECNOLOGÍA \\
\hline Inicial & $\begin{array}{l}\text { Poca o ninguna } \\
\text { intención de usar el } \\
\text { conocimiento. }\end{array}$ & $\begin{array}{l}\text { La organización no } \\
\text { es consciente de la } \\
\text { necesidad de GC. }\end{array}$ & $\begin{array}{l}\text { No existen procesos } \\
\text { formales para capturar, } \\
\text { compartir y reutilizar el } \\
\text { conocimiento. }\end{array}$ & $\begin{array}{l}\text { Sin tecnologías o } \\
\text { infraestructura de soporte } \\
\text { u apoyo. }\end{array}$ \\
\hline Conciencia & $\begin{array}{l}\text { La organización es } \\
\text { consciente y tiene la } \\
\text { intención de GC, pero } \\
\text { posiblemente no sepa } \\
\text { cómo. }\end{array}$ & $\begin{array}{l}\text { La organización está } \\
\text { consciente de la } \\
\text { necesidad de GC. }\end{array}$ & $\begin{array}{l}\text { Se documentan } \\
\text { los conocimientos } \\
\text { indispensables para la } \\
\text { realización de tareas } \\
\text { respectivas. }\end{array}$ & $\begin{array}{l}\text { Se han iniciado proyectos } \\
\text { piloto de GC (no } \\
\text { necesariamente por iniciativa } \\
\text { de directivos). }\end{array}$ \\
\hline Definido & $\begin{array}{l}\text { La organización ha } \\
\text { puesto en marcha } \\
\text { una infraestructura } \\
\text { básica que soporta } \\
\text { la GC. }\end{array}$ & $\begin{array}{l}\text { - Se proporciona } \\
\text { formación básica } \\
\text { sobre GC. } \\
\text { - Se han activado } \\
\text { los sistemas de } \\
\text { incentivos. }\end{array}$ & $\begin{array}{l}\text { - Se han formalizado los } \\
\text { procesos para la gestión } \\
\text { de contenidos } \\
\text { e información. } \\
\text { - Los sistemas de medición } \\
\text { pueden ser usados para } \\
\text { medir el incremento de la } \\
\text { productividad por causa } \\
\text { de GC. }\end{array}$ & $\begin{array}{l}\text { - Se tiene instalada una } \\
\text { infraestructura básica de } \\
\text { GC (páginas amarillas). } \\
\text { Se han puesto en marcha } \\
\text { algunos proyectos de GC } \\
\text { en algunos niveles de la } \\
\text { pirámide organizacional. }\end{array}$ \\
\hline $\begin{array}{l}\text { Gestionado / } \\
\text { establecido }\end{array}$ & $\begin{array}{l}\text { Las iniciativas de GC } \\
\text { están plenamente } \\
\text { establecidas en la } \\
\text { organización. }\end{array}$ & $\begin{array}{l}\text { - La GC está } \\
\text { normalizada a } \\
\text { lo largo de la } \\
\text { organización. } \\
\text { - Formación } \\
\text { avanzada en GC. } \\
\text { - Estándares } \\
\text { organizacionales. }\end{array}$ & $\begin{array}{l}\text { Medición cuantitativa de los } \\
\text { procesos de GC (es decir, } \\
\text { el uso de métricas). }\end{array}$ & $\begin{array}{l}\text { - En toda la organización } \\
\text { los sistemas de GC están } \\
\text { funcionando plenamente. } \\
\text { El uso de los sistemas } \\
\text { de GC está en un nivel } \\
\text { razonable. } \\
\text { - Perfecta integración } \\
\text { de la tecnología con la } \\
\text { arquitectura de contenido. }\end{array}$ \\
\hline Optimizado & $\begin{array}{l}\text { La GC está } \\
\text { plenamente integrada } \\
\text { a la organización y } \\
\text { sometida a procesos } \\
\text { de mejoramiento } \\
\text { continuo }\end{array}$ & $\begin{array}{l}\text { La cultura de } \\
\text { compartir está } \\
\text { institucionalizada }\end{array}$ & $\begin{array}{l}\text { - Los procesos de } \\
\text { GC son revisados } \\
\text { permanentemente y } \\
\text { mejorados. } \\
\text { Los actuales procesos de } \\
\text { GC pueden ser fácilmente } \\
\text { adaptados para satisfacer } \\
\text { las nuevas necesidades. } \\
\text { Los procedimientos de GC } \\
\text { forman parte integral de la } \\
\text { organización. }\end{array}$ & $\begin{array}{l}\text { La infraestructura actual } \\
\text { de GC es mejorada } \\
\text { continuamente. }\end{array}$ \\
\hline
\end{tabular}

Teniendo en cuenta que el modelo G-KMMM es del año 2006 y que dentro de la revisión de la literatura no se encontró alguna actualización del mismo, en este trabajo de investigación se implementó un área clave denominada estrategia que, de acuerdo con [7] permite alinear los esfuerzos de la GC con los focos estratégicos de la organización, principalmente con la innovación (que como se mencionó anteriormente se plantea como uno de los objetivos del SIG de la Unad). Estos mismos autores indican que esta área clave establece 3 capacidades de la GC:
Estrategia de GC. Está relacionada con la caracterización de áreas de conocimiento clave para el presente y el futuro de la organización.

Compromiso de directivos y recursos. Alude a la sostenibilidad de la estrategia de GC en la organización, la cual depende del convencimiento por parte de los líderes sobre los beneficios de la GC y de la asignación de los recursos requeridos para garantizar el despliegue de dicha estrategia a lo largo del tiempo. 
Equipos de GC. Se refiere a la existencia de un equipo que lidera la estrategia de $\mathrm{GC}$ en sus inicios. Una vez implementada la estrategia de GC, este equipo debe desempeñar el papel de facilitador y orientar a los colaboradores para que logren empoderarse de la estrategia de GC.

TABLA III

Estructura del Área Clave de Estrategia

\begin{tabular}{|c|c|}
\hline Descripción & $\begin{array}{l}\text { Permite alinear los esfuerzos de } \\
\text { la GC con los focos estratégicos, } \\
\text { principalmente con la innovación. }\end{array}$ \\
\hline 1. Inicial & $\begin{array}{l}\text { - Existen prácticas informales de GC. } \\
\text { - Hay un compromiso inicial por parte } \\
\text { de líderes con el proceso de GC. } \\
\text { - No existe una persona o equipo que } \\
\text { lidere la GC. }\end{array}$ \\
\hline 2. Conciencia & $\begin{array}{l}\text { - La organización avanza en la } \\
\text { formulación de una definición de GC } \\
\text { que esté articulada con la estrategia. } \\
\text {-El compromiso de los líderes con la } \\
\text { GC conlleva a explorar la asignación } \\
\text { de recursos para su implementación. } \\
\text { - Existe una persona que acompaña } \\
\text { parcialmente la implementación de la GC. }\end{array}$ \\
\hline 3. Definido & $\begin{array}{l}\text { - Existe un vínculo entre la estrategia y } \\
\text { la GC. Se han identificado áreas clave } \\
\text { de conocimiento clave y se desarrollan } \\
\text { prácticas que lo facilitan. } \\
\text { - Los líderes facilitan los recursos } \\
\text { necesarios para la implementación de } \\
\text { las prácticas de GC. } \\
\text { - Se designa un líder con la } \\
\text { responsabilidad de implementar la } \\
\text { estrategia de GC. }\end{array}$ \\
\hline $\begin{array}{l}\text { 4. Gestionado / } \\
\text { establecido }\end{array}$ & $\begin{array}{l}\text { - Se monitorea el impacto de la GC } \\
\text { mediante indicadores y se enfatizan } \\
\text { las prácticas que desarrollan el } \\
\text { conocimiento clave para el presente y } \\
\text { futuro de cara a la estrategia. } \\
\text { - Los líderes acompañan la evolución } \\
\text { de la GC y los indicadores evidencian } \\
\text { sus resultados. } \\
\text { - Se consolida un equipo de trabajo que } \\
\text { lidera la estrategia de GC, sistematiza } \\
\text { y transfiere la experiencia en espacios } \\
\text { internos y externos. }\end{array}$ \\
\hline 5. Optimizado & $\begin{array}{l}\text { - La GC se ha convertido en un proceso } \\
\text { estratégico, clave para todos los } \\
\text { procesos, apalancando la innovación } \\
\text { y la estrategia. } \\
\text { - Los líderes tienen convencimiento } \\
\text { pleno sobre la GC y su impacto sobre } \\
\text { el futuro, lo que conduce a asegurar la } \\
\text { sostenibilidad de la estrategia de GC. } \\
\text { - Los colaboradores comprenden su rol } \\
\text { en la GC y lo ejecutan con autonomía } \\
\text { y compromiso apoyados por el equipo } \\
\text { de la GC. }\end{array}$ \\
\hline
\end{tabular}

En la tercera etapa denominada Aplicación del $M M G C$ se realizó la medición de la madurez de la GC. Una vez seleccionado el Modelo de madurez G-KMMM [1] para la realización del diagnóstico de madurez de GC en la ECBTI, se procedió a su aplicación en la población de esta Escuela, la cual estaba conformada en el primer semestre de 2016 por 570 personas (Decano, Secretario Académico, 565 docentes y 3 personas de apoyo administrativo).

Para la aplicación práctica del modelo G-KMMM, este propone un instrumento de evaluación denominado KM Assessment Tool (KMAT). La herramienta de evaluación KMAT es una encuesta de diagnóstico (direccionada a colaboradores y directivos) que ayuda a una organización a determinar la eficacia de sus prácticas de GC [22].

El instrumento de evaluación KMAT fue implementado en un formulario de Google y enviado a toda la población de la ECBTI a través de la cuenta de correo institucional, cuyo dominio pertenece a Gmail. Cabe señalar que el instrumento se tradujo al español debido a que el modelo G-KMMM se encuentra disponible en inglés. Posteriormente, las preguntas traducidas se adaptaron al contexto de la ECBTI de la Unad. El instrumento de evaluación KMAT enviado, estaba compuesto por un total de 37 preguntas: 13 para el área clave personas, 6 para el área clave procesos, 6 para el área clave tecnología y 12 para el área clave estrategia.

Para que la población de la ECBTI respondiera el instrumento se empleó la escala de Likert con el objetivo de que las personas expresaran su grado de aprobación o desaprobación en cada pregunta a partir de las siguientes opciones: totalmente de acuerdo, de acuerdo, en desacuerdo, totalmente en desacuerdo y no sabe / no responde. Se realizó una prueba piloto del formulario para su validación con 10 profesionales de la ECBTI: 4 docentes ocasionales tiempo completo, 1 docente ocasional medio tiempo, 1 docente ocasional hora cátedra, 2 líderes de programa, 1 docente de planta y 1 persona del cuerpo administrativo. Estos profesionales diligenciaron el instrumento KMAT y lo realimentaron para su posterior aplicación a la población de la Escuela. 
Hasta la fecha en la que el instrumento estuvo habilitado, se lograron un total de 307 encuestas diligenciadas. En este sentido, a través de la muestra alcanzada se obtuvo un nivel de confianza del $96.2 \%$ y un porcentaje de error del $3.8 \%$. Cabe señalar que el nivel de confianza se refiere al nivel de certeza de los resultados y el margen de error está relacionado con el monto de error que el resultado obtenido debería tener [23].

El análisis realizado con los datos obtenidos mediante el instrumento de evaluación KMAT, consiste principalmente en las estadísticas descriptivas para cada pregunta; en este sentido, los resultados se presentan en relación con el porcentaje de respuesta de las opciones positivas (totalmente de acuerdo y de acuerdo), el porcentaje de respuestas negativas (en desacuerdo y totalmente en desacuerdo) y el porcentaje de respuestas "no sabe / no responde".

Teniendo en cuenta, que de acuerdo con el instrumento de evaluación KMAT, en cada área clave se propone más de una pregunta para algunos niveles, se definió asignar una ponderación para las preguntas.

Posteriormente, a partir del nivel que tuvo el mayor porcentaje de las respuestas positivas (totalmente de acuerdo y de acuerdo), se estableció el nivel de madurez para cada área clave, siguiendo el análisis de resultados del artículo de [18].

\section{Resultados y Análisis}

En relación con el área clave personas, en la tabla 4 se presentan los porcentajes obtenidos en cada una de las opciones de respuesta. Con base en estos porcentajes se elaboró una gráfica de tipo radar para identificar el nivel de madurez de esta área clave (Fig. 1). A partir de ella, se infiere que el mayor porcentaje de respuestas positivas (totalmente de acuerdo y de acuerdo) se presentó en el nivel 2 (conciencia), lo que significa que este es el nivel de madurez del área clave personas. En este orden de ideas la ECBTI de la Unad es consciente de la necesidad de la GC para el desarrollo de sus procesos académicos, la investigación y la formación de los estudiantes en sus programas de pregrado y posgrado.

TABLA IV

Porcentajes de Respuesta del Área Clave Personas

\begin{tabular}{|l|c|c|c|}
\hline \multicolumn{1}{|c|}{$\begin{array}{c}\text { NIVEL DE } \\
\text { MADUREZ }\end{array}$} & $\begin{array}{c}\text { \% DE TA } \\
\text { Y DA }\end{array}$ & $\begin{array}{c}\text { \% DE TD } \\
\text { Y D }\end{array}$ & \% DE NS/NR \\
\hline $\begin{array}{l}\text { Nivel 2 } \\
\text { Conciencia }\end{array}$ & $89 \%$ & $8 \%$ & $3 \%$ \\
\hline $\begin{array}{l}\text { Nivel 3 } \\
\text { Definido }\end{array}$ & $45 \%$ & $30 \%$ & $25 \%$ \\
\hline $\begin{array}{l}\text { Nivel 4 } \\
\text { Gestionado }\end{array}$ & $35 \%$ & $35 \%$ & $30 \%$ \\
\hline $\begin{array}{l}\text { Nivel 5 } \\
\text { Optimizado }\end{array}$ & $46 \%$ & $31 \%$ & $23 \%$ \\
\hline
\end{tabular}

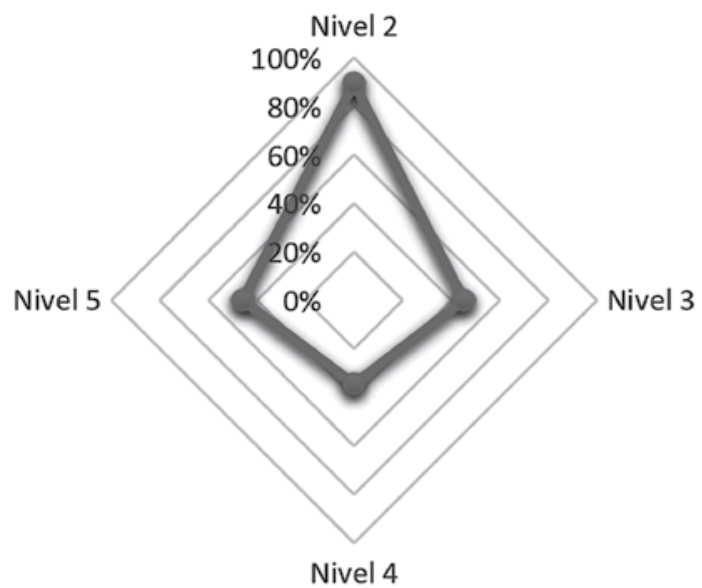

Fig. 1 Nivel de madurez del área clave personas

En cuanto al área clave procesos, en la tabla 5 se presentan los porcentajes obtenidos en cada una de las opciones de respuesta y en la Fig. 2, el nivel de madurez de esta área clave. A partir de los resultados que se presentan en la tabla y la figura es posible indicar que el nivel de madurez de GC del área clave procesos es 3 (definido); en este sentido, se infiere que en la ECBTI se han formalizado los procesos 
para la gestión de contenidos, al igual que el proceso para la recolección e intercambio de información.

TABLA V

Porcentajes de Respuesta del Área Clave Procesos

\begin{tabular}{|l|c|c|c|}
\hline \multicolumn{1}{|c|}{$\begin{array}{l}\text { NIVEL DE } \\
\text { MADUREZ }\end{array}$} & $\begin{array}{c}\text { \% DE TA } \\
\text { Y DA }\end{array}$ & $\begin{array}{c}\text { \% DE TD } \\
\text { Y D }\end{array}$ & \% DE NS/NR \\
\hline $\begin{array}{l}\text { Nivel 2 } \\
\text { Conciencia }\end{array}$ & $64 \%$ & $23 \%$ & $12 \%$ \\
\hline $\begin{array}{l}\text { Nivel 3 } \\
\text { Definido }\end{array}$ & $69 \%$ & $15 \%$ & $15 \%$ \\
\hline $\begin{array}{l}\text { Nivel 4 } \\
\text { Gestionado }\end{array}$ & $39 \%$ & $29 \%$ & $31 \%$ \\
\hline $\begin{array}{l}\text { Nivel 5 } \\
\text { Optimizado }\end{array}$ & $64 \%$ & $12 \%$ & $24 \%$ \\
\hline
\end{tabular}

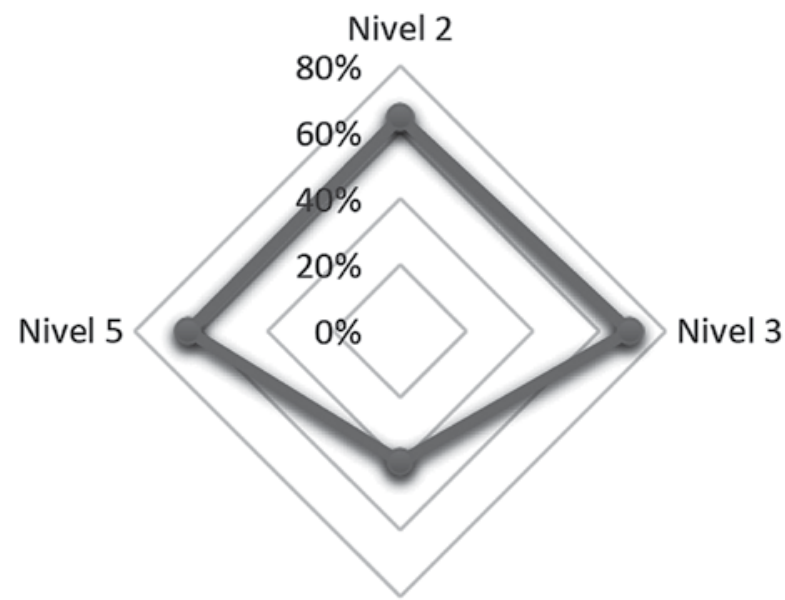

Nivel 4

Fig. 2 Nivel de madurez del área clave procesos

Con respecto al área clave tecnología, los porcentajes de respuesta se presentan en la tabla 6 y el nivel de madurez en la Fig. 3. De acuerdo con dicha información, el nivel de madurez del área clave tecnología es mucho más cercano al 5 (optimizado), teniendo en cuenta que este nivel obtuvo el mayor porcentaje de respuestas positivas $(59 \%)$. Esto significa que la infraestructura que se emplea para la GC en la ECBTI es mejorada de manera continua.
TABLA VI

Porcentajes de Respuesta del Área Clave Tecnología

\begin{tabular}{|l|c|c|c|}
\hline \multicolumn{1}{|c|}{$\begin{array}{l}\text { NIVEL DE } \\
\text { MADUREZ }\end{array}$} & $\begin{array}{c}\text { \% DE TA } \\
\text { Y DA }\end{array}$ & $\begin{array}{c}\text { \% DE TD } \\
\text { Y D }\end{array}$ & \% DE NS/NR \\
\hline $\begin{array}{l}\text { Nivel 2 } \\
\text { Conciencia }\end{array}$ & $58 \%$ & $11 \%$ & $31 \%$ \\
\hline $\begin{array}{l}\text { Nivel 3 } \\
\text { Definido }\end{array}$ & $36 \%$ & $44 \%$ & $19 \%$ \\
\hline $\begin{array}{l}\text { Nivel 4 } \\
\text { Gestionado }\end{array}$ & $54 \%$ & $19 \%$ & $27 \%$ \\
\hline $\begin{array}{l}\text { Nivel 5 } \\
\text { Optimizado }\end{array}$ & $59 \%$ & $18 \%$ & $23 \%$ \\
\hline
\end{tabular}

Nivel 2

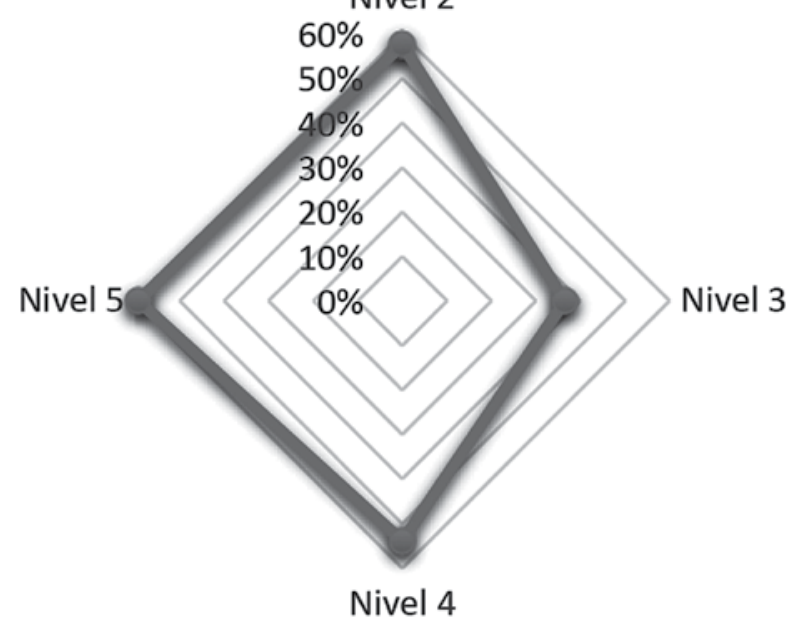

Fig. 3 Nivel de madurez del área clave tecnología

Para el área clave estrategia, en la tabla 7 y en la Fig. 4 se presentan los porcentajes de respuesta y el nivel de madurez, respectivamente. Tomando como referencia dicha figura, es posible establecer que el nivel de madurez de GC del área clave estrategia es 5 (optimizado). En esta medida, la GC se ha convertido en un proceso estratégico para la ECBTI, clave para los procesos tanto de gestión académica como de formación. Adicionalmente, se puede inferir que los líderes académicos de esta Escuela tienen convencimiento sobre la GC y su impacto sobre el futuro. 
TABLA VII

Porcentajes de Respuesta del Área Clave Estrategia

\begin{tabular}{|l|c|c|c|}
\hline $\begin{array}{c}\text { NIVEL DE } \\
\text { MADUREZ }\end{array}$ & $\begin{array}{c}\text { \% DE TA } \\
\text { Y DA }\end{array}$ & $\begin{array}{c}\text { \% DE TD } \\
\text { Y D }\end{array}$ & \% DE NS/NR \\
\hline $\begin{array}{l}\text { Nivel 2 } \\
\text { Conciencia }\end{array}$ & $48 \%$ & $18 \%$ & $34 \%$ \\
\hline $\begin{array}{l}\text { Nivel 3 } \\
\text { Definido }\end{array}$ & $38 \%$ & $19 \%$ & $44 \%$ \\
\hline $\begin{array}{l}\text { Nivel 4 } \\
\text { Gestionado }\end{array}$ & $43 \%$ & $17 \%$ & $39 \%$ \\
\hline $\begin{array}{l}\text { Nivel 5 } \\
\text { Optimizado }\end{array}$ & $57 \%$ & $17 \%$ & $26 \%$ \\
\hline
\end{tabular}

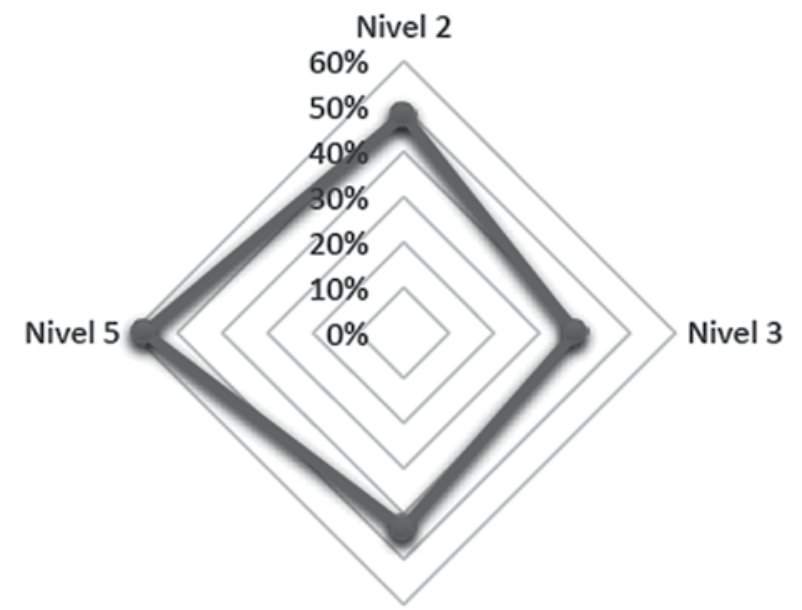

Nivel 4

Fig. 4 Nivel de madurez del área clave estrategia

Una vez identificados los niveles de madurez de GC para cada una de las áreas clave, se procedió a establecer el nivel global de la ECBTI. Para este propósito se asignó una ponderación a cada área clave. Dicha ponderación se presenta en la tabla 8.
TABLA VIII

Ponderación de los Niveles Obtenidos en Cada Área Clave

\begin{tabular}{|l|c|c|c|}
\hline $\begin{array}{l}\text { ÁREA } \\
\text { CLAVE }\end{array}$ & PONDERACIÓN & $\begin{array}{c}\text { NIVELES DE } \\
\text { MADUREZ }\end{array}$ & $\begin{array}{c}\text { RESULTADO } \\
\text { PONDERACIÓN }\end{array}$ \\
\hline Personas & $25 \%$ & 2 & 0,5 \\
\hline Procesos & $25 \%$ & 3 & 0,75 \\
\hline Tecnología & $25 \%$ & 5 & 1,25 \\
\hline Estrategia & $25 \%$ & 5 & 1,25 \\
\hline Nivel de madurez global & & 3,75 \\
\hline
\end{tabular}

Una vez aplicada la ponderación, se encontró que el nivel de madurez de GC global de la ECBTI es mucho más cercano al 4 (gestionado), lo que significa que las iniciativas de GC están plenamente establecidas en esta Escuela de la UNAD.

Si bien el nivel de madurez de GC global de la ECBTI fue de 4 (gestionado), fueron las áreas clave de tecnología y estrategia las que obtuvieron los mejores resultados, ya que presentaron el máximo nivel de madurez (optimizado). No obstante, las áreas clave de personas y procesos no presentaron resultados tan favorables, ya que obtuvieron niveles de madurez de 2 (conciencia) y 3 (definido), respectivamente.

En el caso del área clave de personas, aunque la ECBTI es consciente de la necesidad de la GC tanto en los procesos de gestión académica como de formación, requiere implementar programas de formación sobre GC e iniciar sistemas de incentivos para que dicha gestión sea desarrollada de manera efectiva por todos los colaboradores de esta Escuela, en aras de alcanzar el siguiente nivel de madurez que es 3 (definido). Posteriormente, para lograr el nivel de madurez 5 (optimizado) en esta área clave, se requiere normalizar la GC y la cultura de compartir en todos los centros de la Unad donde esta Escuela hace presencia.

En materia del área clave de procesos, la ECBTI tiene formalizado el proceso para la gestión de contenidos, al igual que el proceso para la recolección 
e intercambio de información. Sin embargo, para alcanzar el nivel de madurez 4 (gestionado) en esta área clave, es necesario empezar a realizar mediciones de orden cuantitativo de dichos procesos. Para lograr el máximo nivel de madurez en esta área clave (optimizado), es necesario revisar y mejorar permanentemente los procesos de GC, que dichos procesos puedan ser adaptados para satisfacer las nuevas necesidades del servicio educativo y que los procedimientos de GC formen parte integral de la ECBTI.

El nivel de madurez de GC "5 - optimizado" (máximo nivel de madurez de GC que establece el modelo) del área clave de tecnología, significa que la infraestructura tecnológica que emplea la ECBTI para gestionar el conocimiento funciona plenamente, se mejora continuamente y existe una integración de la tecnología con la arquitectura de contenidos. Este nivel identificado se soporta con la robusta infraestructura tecnológica que tiene la Unad para el desarrollo de todos los procesos académicos y administrativos de la Universidad y que se somete a procesos de mejora continua; existe una dependencia que se encarga del proceso de gestión de servicios de infraestructura tecnológica denominada Gerencia de Innovación y Desarrollo Tecnológico (GIDT), la cual evalúa las condiciones técnicas de los recursos tecnológicos de la Universidad, realiza su mantenimiento y actualización de manera preventiva y correctiva y evalúa y propone la incorporación planificada de nuevas y mejores tecnologías.

Con respecto al nivel de madurez de GC " 5 - optimizado" del área clave estrategia, significa que la GC es un proceso estratégico, clave para todos los procesos, apalancando la innovación y la estrategia. Esto se sustenta desde la misión de la ECBTI, ya que se centra en la formación a distancia, mediante estrategias educativas que incluyen el uso de las TIC para formar profesionales integrales, capaces de responder a las necesidades del entorno local, regional e internacional, por medio de la investigación y el desarrollo (I\&D) como factor de desarrollo productivo y social en las comunidades donde impacta. En este orden de ideas, es posible inferir que la ECBTI tiene como eje fundamental la Gestión del Conocimiento, teniendo en cuenta que el propósito de esta en las Instituciones de la Educación Superior consiste en crear, adquirir, procesar, conservar, transmitir y transferir el conocimiento, mediante la articulación de la docencia, la investigación y la extensión con el fin de formar profesionales que posean el conocimiento para atender las necesidades sociales, científicas y técnicas de la sociedad.

El nivel de madurez de GC global "4 - gestionado" identificado en la ECBTI bajo la luz del modelo empleado, significa en términos generales que esta unidad académica misional de la Unad tiene plenamente establecidas las iniciativas para gestionar el conocimiento. No obstante, es necesario que la GC logre estar integrada a lo largo de todos los centros de la Unad donde la ECBTI hace presencia y sea sometida a procesos de mejoramiento continuo para poder alcanzar el máximo nivel de madurez de GC "5 - optimizado".

En consecuencia, el diagnóstico de madurez de GC desarrollado en la Escuela de Ciencias Básicas de Tecnología e Ingeniería, se constituye como el primer paso para mejorar sustantivamente los procesos de GC que se llevan a cabo en la misma; el presente diagnóstico se debe concebir como punto de partida para proponer iniciativas y metas dentro de la ECBTI que permitan generar una cultura efectiva de compartir el conocimiento dentro de toda la comunidad académica y que contribuyan a optimizar los procedimientos que se tienen para gestionar el conocimiento.

Es importante resaltar que el modelo empleado para medir la madurez de Gestión del Conocimiento "G-KMMM" permitió cumplir el objetivo principal de este trabajo de investigación aplicada. Gracias a este modelo, fue posible realizar de manera exitosa la medición de madurez de GC por cada área clave, para posteriormente establecer el nivel de madurez de GC global en la ECBTI. De igual forma, el área clave estrategia implementada para complementar el modelo, se podría concebir como un aporte significativo en lo que a modelos de madurez de GC se refiere, ya que mediante esta es posible identificar la articulación que existe entre la Gestión del Conocimiento y los focos estratégicos de la organización. En el caso 
de la ECBTI, dicha articulación conllevó a establecer que la GC es un proceso estratégico clave para los procesos tanto académicos como administrativos que emprende esta unidad misional de la Unad.

En este sentido, es posible concluir que el Modelo general de madurez de GC (G-KMMMM) [1] es muy apropiado para emplearse en Instituciones de Educación Superior de gran complejidad (como lo es el caso de la Unad), ya que efectivamente propone una metodología muy detallada en su aplicación y análisis y un instrumento de medición (KMAT) de fácil comprensión para todos los colaboradores de cualquier tipo de organización.

\section{Conclusiones y Trabajos Futuros}

En el presente artículo se ha presentado una revisión sistemática sobre los modelos de madurez de Gestión del Conocimiento, a partir de la cual fue posible seleccionar y adaptar el mejor para medir la madurez de GC en la ECBTI, de la UNAD. El modelo elegido fue el General Knowledge Management Maturity Model (G-KMMM) [1], el cual se adaptó, adicionando un área clave: estrategia.

Se aplicó el instrumento ajustado a 307 de los 570 docentes y funcionarios de la ECBTI, logrando identificar el nivel de madurez de GC tanto global como por áreas clave de esta Escuela de la UNAD y los resultados fueron analizados siguiendo a [18], [20] y [7].

La información recopilada se analizó, encontrando que las áreas clave de personas y procesos no presentaron resultados muy favorables, ya que obtuvieron niveles de madurez de 2 (conciencia) y 3 (definido), respectivamente. En otras palabras, la ECBTI de la Unad es consciente de la necesidad de la GC para el desarrollo de sus procesos académicos, la investigación y la formación de los estudiantes en sus programas de pregrado y posgrado; así mismo, se han formalizado los procesos para la gestión de contenidos, al igual que el proceso para la recolección e intercambio de información.
Por su parte, las áreas clave de tecnología y estrategia obtuvieron los mejores resultados, alcanzando el nivel 5 (optimizado) en ambos casos. Esto quiere decir que la infraestructura que se emplea para la GC en la ECBTI se mejora de manera continua y que sus líderes académicos están convencidos de la GC y de su impacto sobre el futuro.

Todo lo anterior, permitió establecer que el nivel de madurez de GC global de la ECBTI fue de 4 (gestionado). Aunque se requiere trabajo, especialmente en las áreas de personas y procesos, las iniciativas de GC están plenamente establecidas en esta Escuela de la Unad.

Los hallazgos permitieron establecer algunas recomendaciones, las cuales se enuncian a continuación:

- Se requiere implementar programas de formación sobre GC.

- Es necesario proponer sistemas de incentivos para que todos los colaboradores de la Escuela desarrollen de manera efectiva la GC.

- Se requieren medios y canales de comunicación eficaces que propendan por facilitar y compartir el conocimiento que se crea al interior de la Escuela en los diferentes puntos del territorio.

- Se requiere implementar mediciones cuantitativas asociadas a los procesos de GC.

Asimismo, para alcanzar con el tiempo el nivel 5 (optimizado) en las áreas clave de personas y procesos, es necesario emprender las siguientes acciones en la ECBTI:

- Normalizar la GC y la cultura de compartir en todos los centros de la Unad donde esta Escuela hace presencia.

- Revisar y mejorar permanentemente los procesos de GC.

- Lograr la adaptabilidad de los procesos para satisfacer las nuevas necesidades del servicio educativo.

- Conseguir que los procedimientos de GC formen parte integral de la ECBTI. 
Con respecto a las limitaciones, cabe mencionar que la distribución tan dispersa de los docentes en todo el territorio nacional, dificultó el diligenciamiento del instrumento por parte de toda la población de la ECBTI. Si bien se emprendieron actividades concretas para lograr el diligenciamiento total del instrumento en esta Escuela, como por ejemplo, su envío desde la cuenta de correo electrónico oficial de la ECBTI y la gestión directa con los docentes por medio de los líderes de programa, líderes zonales y directores de los cursos virtuales, solo 307 profesionales diligenciaron la encuesta, lo que equivale al $54 \%$ del total de personas que la conforman.

Dentro de los futuros trabajos se encuentra la medición de la madurez de Gestión del Conocimiento en toda la Unad y un trabajo comparativo con otras instituciones de educación superior.

\section{ReFERENCIAS}

[1] H. Y. Teah, L. G. Pee and A. Kankanhalli, "Development and application of a general knowledge management maturity model," Tenth Pacific Asia Conf. Inf. Syst. (PACIS 2006), no. 12, 2006, pp. 401-416,.

[2] UNAD, "Estatuto Organizacional de la Universidad Nacional Abierta y a Distancia (UNAD)," Bogotá D.C., 2012.

[3] UNAD, "Reglamento Estudiantil de la Universidad Nacional Abierta y a Distancia," Bogotá D.C., 2013.

[4] UNAD, "Sistema Integrado de Gestión (SIG) de la Universidad Nacional Abierta y a Distancia," 2014. [Online]. Available: http://sig.unad.edu.co/.

[5] UNAD, "Escuela de Ciencias Básicas, Tecnología e Ingeniería de la Universidad Nacional Abierta y a Distancia," 2012. [Online]. Available: http://estudios.unad.edu.co/ecbti.

[6] M. Gómez-Zermeño y L. Alemán, Administración de proyectos de capacitación basados en tecnología. Monterrey, México: Editorial Digital del Tecnológico de Monterrey, 2012.

[7] J. Arias-Pérez, J. Tavera-Mesías y D. Castaño-Serna, "Construcción de un modelo de madurez de gestión del conocimiento para una multinacional de alimentos de una economía emergente.," El Prof. la Inf., vol. 25, no. 1, 2016, pp. 88-102.

[8] D. Rodríguez, "Modelos para la creación y gestión del conocimiento: una aproximación teórica," Educar, vol. 37, 2006, pp. 25-39.

[9] E. Serna, "Maturity model of knowledge management in the interpretativist perspective," Int. J. Inf. Manage., vol. 32, no. 4, 2012, pp. 365-371.
[10] R. Escobar, C. Montenegro, W. Joven y J. López, "Modelos de Gestión del Conocimiento que integren tecnologías elearning en la Educación Superior," Redes Ing., vol. 4, 2013, pp. 103-113.

[11] E. Bustos, M. Trinidad, and M. García, "Modelo de gestión de conocimiento para el desarrollo de posgrado: estudio de caso," Rev. Electrónica Investig. Educ., vol. 18, no. 128139, 2016.

[12] A. Pérez, L. Ruiz, C. Varela, F. Grosso, C. Camós, A. Trottini, M. de Lujan, and S. Darin, Gestión del conocimiento. Un nuevo enfoque aplicable a las organizaciones y a la universidad, 1ra ed. Buenos Aires: Norma, 2005.

[13] M. Lopera y N. Quiroz, "Caracterización de un modelo de gestión del conocimiento aplicable a las funciones universitarias de investigación y extensión: Caso universidad Ces," Universidad del Rosario, 2013.

[14] R. Abdullah, M. Selamat, S. Sahibudin and R. Alias, "A framework for knowledge management system implementation in collaborative environment for higher learning institution," J. Knowl. Manag. Pract., vol. 6, no. 1, 2005.

[15] A. Kok, "Intellectual capital management as part of knowledge management initiatives at institutions of higher learning," Electron. J. Knowl. Manag., vol. 5, no. 2, 2007, pp. 181-192.

[16] Y. Yeh, "The Implementation of knowledge management system In Taiwan's higher education," J. Coll. Teach. Learn., vol. 2, no. 9, 2011, pp. 35-42.

[17] J. Flores, "La gestión del conocimiento y las herramientas colaborativas: una alternativa de aplicación en Instituciones de educación superior," Rev. Invest. (Guadalajara)., vol. 34, no. 71, 2010, pp. 11-32.

[18] B. Demchig, "Knowledge Management Capability Level Assessment of the Higher Education Institutions: Case Study from Mongolia," Procedia - Soc. Behav. Sci., vol. 174, 2015, pp. 3633-3640.

[19] G. Klimko, "Knowledge management and maturity models: Building common understanding," in Second European Conference on Knowledge Management: Bled School of Management, Academic Conferences Limited, Ed. Bled, Slovenia, 2001, pp. 269-278.

[20] C. Durango, M. Quintero y C. Ruiz, "Metodología para evaluar la madurez de la gestión del conocimiento en algunas grandes empresas colombianas," Tecnura, vol. 19, no. 43, 2013, pp. 20-36.

[21] C. Durango, "Evaluación de las Tecnologías para la Gestión del Conocimiento," Rev. Dimens. Empres., vol. 13, no. 2, 2015, pp. 205-217.

[22] L. Pee and A. Kankanhalli, "A Model of Organisational Knowledge Management Maturity Based on People, Process, and Technology," J. Inf. Knowl. Manag., vol. 08, no. 02, 2009, pp. 79-99.

[23] Universidad Nacional del Nordeste, "Tamaño de una muestra," Departamento de Sistemas Informáticos Integrales de la Facultad de Medicina, 2016. [Online]. Available: http:// med.unne.edu.ar/biblioteca/calculos/calculadora.htm. 
